\title{
Existence of Random Attractor for Stochastic Fractional Long-Short Wave Equations with Periodic Boundary Condition
}

\author{
Na Liu ${ }^{1}$ and Jie Xin ${ }^{1,2}$ \\ ${ }^{1}$ School of Mathematics and Statistics Science, Ludong University, Yantai City 264025, China \\ ${ }^{2}$ School of Mathematical Sciences, Qufu Normal University, Qufu 273165, China \\ Correspondence should be addressed to Jie Xin; fdxinjie@sina.com
}

Received 30 March 2017; Accepted 8 June 2017; Published 26 July 2017

Academic Editor: Ciprian G. Gal

Copyright (C) $2017 \mathrm{Na}$ Liu and Jie Xin. This is an open access article distributed under the Creative Commons Attribution License, which permits unrestricted use, distribution, and reproduction in any medium, provided the original work is properly cited.

We consider the asymptotic behaviors of stochastic fractional long-short equations driven by a random force. Under a priori estimates in the sense of expectation, using Galerkin approximation by the stopping time and the Borel-Cantelli lemma, we prove the existence and uniqueness of solutions. Then a global random attractor and the existence of a stationary measure are obtained via the Birkhoff ergodic theorem and the Chebyshev inequality.

\section{Introduction}

Long-short wave resonance equations arise in the study of the interaction of the surface waves with both gravity and capillary modes presence and also in the analysis of internal waves, as well as Rossby wave in [1]. In the plasma physics, they describe the resonance of the high-frequency electron plasma oscillation and associated low-frequency ion density perturbation in [2]. Long wave and short wave equations with periodic boundary condition have attracted considerable attention as a result of their rich physical and mathematical properties. Guo [3, 4] proved the existence of global solutions for long-short wave equations and generalized long-short wave equations. The existence of global attractor was studied in [5-9].

The stochastic partial differential equation (SPDE) is a kind of partial differential equation with random term and random coefficients, which come from the random environmental effects and the errors of measurement. SPDE is used to describe better complex phenomenon, for example, quantum field theory, statistical mechanics, and financial mathematics; see [10-12] and so on. In [13-19], the authors obtained the existence and uniqueness of the solution and of attractors for SPDEs.

In this paper, we consider that the random environmental effects and the errors of measurement are included into the model of fractional long-short wave equations. More specifically, we study the following equations:

$$
\begin{aligned}
i u_{t}-(-\Delta)^{\alpha} u-n u+i \delta u & =f+\dot{W}_{1}, \\
n_{t}+\beta n+|u|_{x}^{2} & =g+\dot{W}_{2}
\end{aligned}
$$

with the initial condition

$$
\begin{aligned}
& u(x, 0)=u_{0}(x), \\
& n(x, 0)=n_{0}(x),
\end{aligned}
$$

and periodic boundary condition

$$
\begin{aligned}
& u(x, t)=u(x+2 \pi, t), \\
& n(x, t)=n(x+2 \pi, t)=0,
\end{aligned}
$$

where $x \in D=[0,2 \pi]$ in $\mathbb{R}^{1} ; t \geq \tau \in \mathbb{R}_{+} ; \delta, \beta, \alpha>0$. The unknown complex valued function $u(x, t)$ is short surface wave packet and the unknown real valued function $n(x, t)$ is a long interfacial wave. $W_{1}$ and $W_{2}$ are independent $L^{2}(D)$ value Wiener processes which are from the errors of measurement or the random environmental effects and can be seen in detail in the next section. 
Since the solution $u(x, t)$, if it exists, is a $2 \pi$-periodic function, we have the Fourier expansion

$$
u(x, t)=\sum_{k \in \mathbb{Z}} a_{k}(t) e^{i k x},
$$

where $a_{k}(t): \mathbb{R}^{+} \rightarrow \mathbb{R}, x \in \mathbb{R}$.

Hence,

$$
\partial_{x} u=\sum_{k \in \mathbb{Z}} i k a_{k}(t) e^{i k x},
$$

and $(-\triangle)^{\alpha} u$ is defined by

$$
(-\triangle)^{\alpha} u=\sum_{k \in \mathbb{Z}}|k|^{2 \alpha} a_{k}(t) e^{i k x} .
$$

Since

$$
\begin{aligned}
\left|e^{i r x}\right|^{2} & =1, \\
\int_{D} e^{i r x} d x & =0
\end{aligned}
$$

$\forall r \in \mathbb{R}$,

the following definitions make sense. Let

$$
\begin{aligned}
A & =\left\{\left.u\left|u=\sum_{k \in \mathbb{Z}} a_{k}(t) e^{i k x}, \sum_{k \in \mathbb{Z}}\right| k\right|^{4 \alpha} a_{k}^{2}(t)\right. \\
& \left.<\infty, \sum_{k \in \mathbb{Z}}\left|a_{k}(t)\right|^{2}<\infty\right\},
\end{aligned}
$$

and let $H^{2 \alpha}$ be a complete distance space of the set $A$ under the norm

$$
\begin{aligned}
\|u\|_{H^{2 \alpha}}= & \left(2 \pi \sum_{k \in \mathbb{Z}}|k|^{4 \alpha} a_{k}^{2}(t)\right)^{1 / 2} \\
& +\left(2 \pi \sum_{k \in \mathbb{Z}}\left|a_{k}(t)\right|^{2}\right)^{1 / 2} .
\end{aligned}
$$

It is easy to get it to be a Banach space, and that $\forall u \in H^{2 \alpha}, u$ is space-periodic with the period $2 \pi$ and its $2 \alpha$ order derivatives are in $L_{\text {per }}^{2}(D)$. And $\forall u, v \in H^{\alpha}$,

$$
\begin{gathered}
\frac{d}{d t} u v=u \frac{d}{d t} v+v \frac{d}{d t} u, \\
\left((-\triangle)^{\alpha} u, v\right)=\left((-\triangle)^{\alpha_{1}} u,(-\triangle)^{\alpha_{2}} v\right),
\end{gathered}
$$

when $\alpha_{1}+\alpha_{2}=\alpha, 0 \leq \alpha_{1}, \alpha_{2} \leq \alpha . H^{2 \alpha}$ is a Hilbert space with the inner product

$$
(u, v)_{H^{2 \alpha}}=\left((-\triangle)^{\alpha} u,(-\triangle)^{\alpha} v\right) .
$$

The rest of this paper is arranged as follows. In Section 2, we present some preliminaries results. In Section 3, we give a series of time uniform a priori estimates in different energy spaces which will be used to prove our main results; see [20]. In Section 4, we show the existence and uniqueness of solutions for (1)-(2). In Section 5, the random weak attractor and the stationary measure are constructed.

\section{Preliminary}

In this paper, we outline the variational framework for studying problems (1)-(2) and list some preliminary lemmas which will be used later.

We define a complete probability space $\left(\Omega, \mathscr{F},\left\{\mathscr{F}_{t}\right\}_{t \geq 0}\right.$, $\mathbb{P})$. $\mathbb{E}$ denotes the expectation operator with respect to $\mathbb{P}$. Stochastic terms $W_{1}(t)$ and $W_{2}(t)$ are defined on $\left(\Omega, \mathscr{F},\left\{\mathscr{F}_{t}\right\}_{t \geq 0}, \mathbb{P}\right)$ by

$$
\begin{aligned}
& W_{1}(t)=q_{1}(x) \omega_{1}(t), \\
& W_{2}(t)=q_{2}(x) \omega_{2}(t),
\end{aligned}
$$

where $\omega_{1}(t)$ is a standard complex valued Wiener process, $\omega_{2}(t)$ is a standard real valued Winer process independent of $\omega_{1}(t)$, and $q_{1}(x), q_{2}(x)$ are sufficiently smooth functions in a manner. The different inner product spaces for the solution $(u, n)$ of (1)-(2) are defined as

$$
\begin{aligned}
V_{0}= & H_{0}^{\alpha}(D) \times L^{2}(D), \\
V_{1}= & \left(H^{2 \alpha}(D) \cap H_{0}^{\alpha}(D)\right) \times H_{0}^{1}(D), \\
V_{2}= & \left\{\varphi \in H^{3 \alpha}(D) \cap H_{0}^{\alpha}(D):(-\Delta)^{\alpha} \varphi \in H_{0}^{\alpha}(D)\right\} \\
& \times\left(H^{2}(D) \cap H_{0}^{1}(D)\right) .
\end{aligned}
$$

Endow each $V_{i}(i=0,1,2)$ with the usual norm, and satisfy $V_{2} \subset V_{1} \subset V_{0}$ with compact embedding.

Let $\left(\mathscr{X},\|\cdot\|_{\mathscr{X}}\right) \subset(\mathscr{Y},\|\cdot\| \mathscr{Y}) \subset(\mathscr{E},\|\cdot\| \mathscr{Z})$ be three Banach reflective spaces which satisfy $\mathscr{X} \subset \mathscr{Y}$ with compact and dense embedding. The Banach space can be defined as follows:

$$
\mathscr{G}=\left\{v: v \in L^{2}(0, T ; \mathscr{X}), \frac{d v}{d t} \in L^{2}(0, T ; \mathscr{Z})\right\}
$$

endowed with the natural norm

$$
\|v\|_{\mathscr{G}}^{2}=\int_{0}^{T}\|v\| \mathscr{X} d s+\int_{0}^{T}\left\|\frac{d v}{d t}\right\|^{2} \mathscr{Z} d s, \quad v \in \mathscr{G} .
$$

We obtain the following lemma with regard to compactness result by [21].

Lemma 1. If $K$ is bounded in $\mathscr{G}$, then $K$ is precompact in $L^{2}(0, T ; \mathcal{Y})$.

In order to get maximal estimates on stochastic integrals, we need another lemma. $U$ and $H$ are Hilbert spaces which are separable and $W$ is a $Q-W i e n e r$ process on $U_{0}$ with $U_{0}=$ $Q^{1 / 2}$. Let $L_{2}^{0}=L_{2}^{0}\left(U_{0}, H\right)$ be the space of Hilbert-Schmidt operators from $U_{0}$ to $H$. For such operators, we obtain the following lemma by [22]. 
Lemma 2. For any $r \geq 1$ and any $L_{2}^{0}$-valued predictable process $\Phi(t), t \in[0, T]$, we get

$$
\begin{aligned}
& \mathbb{E} \int_{0}^{T} \Phi(s) d W(s)=0, \\
& \mathbb{E}\left(\sup \left|\int_{0}^{s} \Phi(\sigma) d W(\sigma)\right|^{2 r}\right) \\
& \leq c_{r} \sup \mathbb{E}\left(\left|\int_{0}^{s} \Phi(\sigma) d W(\sigma)\right|^{2 r}\right) \\
& \leq C_{r} \mathbb{E}\left(\int_{0}^{t}\|\Phi(s)\|_{L_{2}^{0}}^{2}\right),
\end{aligned}
$$

where $c_{r}$ and $C_{r}$ are some positive constants dependent on $r$.

\section{Uniform A Priori Estimates in Time}

\subsection{A Priori Estimates in $V_{0}$}

Lemma 3. Provided that $u_{0}, f, q_{1} \in L^{2}(D)$, then for any $T>$ 0 and $p \geq 1$, we obtain $u \in L^{2 p}\left(\Omega ; L^{\infty}\left([0, T] ; L^{2}(D)\right)\right) \cap$ $L^{\infty}\left([0, \infty) ; L^{2 p}\left(\Omega ; L^{2}(D)\right)\right)$.

Proof. Taking the inner product of (1) with $2 u$, we get

$$
\left(i u_{t}-(-\Delta)^{\alpha} u-n u+i \delta u, 2 u\right)=\left(f+\dot{W}_{1}, 2 u\right) .
$$

Taking the imaginary part of (19) and applying the Itô formula to $\|u\|^{2}$, one gets

$$
\begin{aligned}
\frac{d}{d t}\|u\|^{2}= & -2 \delta\|u\|^{2}+2 \operatorname{Im} \int_{D} f \bar{u} d x \\
& +2 \operatorname{Im} \int_{D} \bar{u} \dot{W}_{1} d x+\left\|q_{1}\right\|^{2}
\end{aligned}
$$

By (20), using Hölder's and Young's inequalities, we can obtain

$$
\frac{d}{d t}\|u\|^{2}+\delta\|u\|^{2} \leq \frac{1}{\delta}\|f\|^{2}+\left\|q_{1}\right\|^{2}+2 \operatorname{Im} \int_{D} \bar{u} \dot{W}_{1} d x
$$

On the one hand, multiplying by $e^{\delta t}$ and integrating from 0 to $t$ on both sides of (21), we get

$$
\begin{aligned}
& \mathbb{E}\|u\|^{2} \leq e^{-\delta t} \mathbb{E}\left\|u_{0}\right\|^{2}+\frac{1}{\delta^{2}}\|f\|^{2}+\frac{1}{\delta}\left\|q_{1}\right\|^{2} \leq C, \\
& t>0 \text {, }
\end{aligned}
$$

where $C$ is independent of $T$.

On the other hand, integrating from 0 to $t$ and taking the supremum and the expectation on both sides of (21), we can obtain

$$
\begin{aligned}
\sup _{0 \leq t \leq T}\|u\|^{2} \leq & \mathbb{E}\left\|u_{0}\right\|^{2}+\left(\frac{1}{\delta}\|f\|^{2}+\left\|q_{1}\right\|^{2}\right) T \\
& +\mathbb{E} \sup _{0 \leq t \leq T}\left|\int_{0}^{t} \operatorname{Im} \int_{D} \bar{u} \dot{W}_{1} d x d s\right|^{2}+1 .
\end{aligned}
$$

By Lemma 2, for any positive constant $C$, we obtain

$$
\mathbb{E} \sup _{0 \leq t \leq T}\left|\int_{0}^{t} \operatorname{Im} \int_{D} \bar{u} \dot{W}_{1} d x d s\right|^{2} \leq C\left\|q_{1}\right\|^{2} \mathbb{E} \int_{0}^{T}\|u\|^{2} d s .
$$

From (24), for any $T>0$, there exists a positive constant $C_{T}$ such that

$$
\begin{aligned}
\sup _{0 \leq t \leq T}\|u\|^{2} \leq & \mathbb{E}\left\|u_{0}\right\|^{2}+\left(\frac{1}{\delta}\|f\|^{2}+\left\|q_{1}\right\|^{2}\right) T \\
& +C\left\|q_{1}\right\|^{2} \mathbb{E} \int_{0}^{T}\|u\|^{2} d s+1 \\
\leq & C_{T}\left(\mathbb{E}\left\|u_{0}\right\|^{4}+\|f\|^{4}+\left\|q_{1}\right\|^{4}+1\right) .
\end{aligned}
$$

On the basis of the above estimates, we can further give an estimate of $\|u(t)\|_{0}^{2 p}$ for any $p \geq 1$. Applying the Itô formula and Hölder's inequality, we obtain

$$
\begin{aligned}
\frac{d}{d t}\|u\|^{2 p} \leq & -\frac{\delta p}{2}\|u\|^{2 p}+c\left(\|f\|^{2 p}+\left\|q_{1}\right\|^{2 p}\right) \\
& +2 p\|u\|^{2(p-1)} \operatorname{Im} \int_{D} \bar{u} \dot{W}_{1} d x .
\end{aligned}
$$

On the one hand, multiplying by $e^{(\delta p / 2) t}$, integrating from 0 to $t$, and taking the expectation on both sides of (26), we can have

$$
\begin{array}{r}
\mathbb{E}\|u\|^{2 p} \leq e^{-(\delta p / 2) t} \mathbb{E}\left\|u_{0}\right\|^{2 p}+c\left(\|f\|^{2 p}+\left\|q_{1}\right\|^{2 p}\right) \leq C, \\
t>0,
\end{array}
$$

where $C$ is independent of $T$.

On the other hand, by (26), we have

$$
\begin{aligned}
\frac{d}{d t}\|u\|^{2 p} \leq & c\left(\|f\|^{2 p}+\left\|q_{1}\right\|^{2 p}\right) \\
& +2 p\|u\|^{2 p-1} \operatorname{Im} \int_{D} \bar{u} \dot{W}_{1} d x .
\end{aligned}
$$

Integrating from 0 to $t$ and taking the supremum and the expectation on both sides of (28), we get

$$
\begin{aligned}
& \mathbb{E}_{0 \leq t \leq T}\|u\|^{2 p} \\
& \leq \mathbb{E}\|u(0)\|^{2 p}+c\left(\|f\|^{2 p}+\left\|q_{1}\right\|^{2 p}\right) T+1 \\
& \quad+\mathbb{E} \sup _{0 \leq t \leq T}\left|\int_{0}^{t} p\|u\|^{2(p-1)} \operatorname{Im} \int_{D} \bar{u} \dot{W}_{1} d x d s\right|^{2} .
\end{aligned}
$$

By Lemma 2, for any positive constant $C$, we have

$$
\begin{aligned}
& \mathbb{E} \sup _{0 \leq t \leq T}\left|\int_{0}^{t} p\|u\|^{2(p-1)} \operatorname{Im} \int_{D} \bar{u} \dot{W}_{1} d x d s\right|^{2} \\
& \leq C p^{2}\left\|q_{1}\right\|^{2} \mathbb{E} \int_{0}^{T}\|u\|^{4 p-2} d s,
\end{aligned}
$$


where $2 p-1 \geq 1$. Then inserting (30) into (29), and by (27), for any $T>0$, we obtain

$$
\mathbb{E} \sup _{0 \leq t \leq T}\|u\|^{2 p} \leq C_{T}\left(u_{0}, f, q_{1}\right),
$$

where $C_{T}\left(u_{0}, f, q_{1}\right)$ is a positive constant depending on $u_{0}, f, q_{1}$, and $T$.

Lemma 4. Provided that $\left(u_{0}, n_{0}\right) \in V_{0}, q_{1} \in H_{0}^{\alpha}(D), q_{2} \in$ $L^{2}(D), f, g \in L^{2}(D)$, then for any $T>0$ and $p \geq 1$, we have $(u, n) \in L^{\infty}\left((0, \infty) ; L^{2 p}\left(\Omega ; V_{0}\right)\right) \cap L^{2 p}\left(\Omega ; L^{\infty}\left(0, T ; V_{0}\right)\right)$.

Proof. Taking the inner product of (2) with $2 n$, we have

$$
\left(n_{t}+\beta n+|u|_{x}^{2}, 2 n\right)=\left(g+\dot{W}_{2}, 2 n\right) .
$$

Applying the Itô formula to $\|n\|^{2}$, since $n_{t}=-|u|_{x}^{2}-\beta n+$ $g+\dot{W}_{2}$, we get

$$
\begin{gathered}
\frac{d}{d t}\|n\|^{2}-\left\|q_{2}\right\|^{2}+2 \int_{D} n|u|_{x}^{2} d x+2 \beta\|n\|^{2} \\
-2 \int_{D} g n d x-2 \int_{D} n \dot{W}_{2} d x=0 .
\end{gathered}
$$

From (1), we get that

$$
\begin{aligned}
& \int_{D} n|u|_{x}^{2} d x=2 \operatorname{Re} \int_{D} \bar{u}_{x}(u n) d x \\
& =-2 \operatorname{Re} \int_{D} \bar{u}_{x}\left(-i u_{t}+(-\Delta)^{\alpha} u-i \delta u+f-\dot{W}_{1}\right) d x \\
& =\int_{D} i \delta\left(u_{x} \bar{u}-\bar{u}_{x} u\right) d x-2 \operatorname{Re} \int_{D} u \bar{f}_{x} d x \\
& \quad-2 \operatorname{Re} \int_{D} u \overline{\dot{W}}_{1 x} d x .
\end{aligned}
$$

Notice that

$$
\begin{aligned}
& \frac{d}{d t} \int_{D} i\left(u_{x} \bar{u}-u \bar{u}_{x}\right) d x \\
& \quad=2 \int_{D} i\left(u_{x} \bar{u}_{t}-u_{t} \bar{u}_{x}\right) d x+2 \operatorname{Im} \int_{D} q_{1} \bar{q}_{1 x} d x .
\end{aligned}
$$

From (33), (34), and (35), we can have

$$
\begin{aligned}
\frac{d}{d t}( & \left.\|n\|^{2}+\int_{D} i\left(u_{x} \bar{u}-u \bar{u}_{x}\right) d x\right)+2 \beta\|n\|^{2} \\
= & -2 \int_{D} i \delta\left(u_{x} \bar{u}-\bar{u}_{x} u\right) d x+4 \operatorname{Re} \int_{D} u \bar{f}_{x} d x \\
& +2 \operatorname{Im} \int_{D} q_{1} \bar{q}_{1 x} d x+\left\|q_{2}\right\|^{2}+4 \operatorname{Re} \int_{D} u \bar{W}_{1 x} d x \\
& +2 \int_{D} g n d x+2 \int_{D} n \dot{W}_{2} d x .
\end{aligned}
$$

Applying the Itô formula to $\left\|u_{x}\right\|^{2}$, and taking the inner product of (1) with $\left(u_{t}+\delta u\right)$, we obtain

$$
\begin{aligned}
& \left(i u_{t}-(-\Delta)^{\alpha} u-n u+i \delta u,\left(u_{t}+\delta u\right)\right) \\
& =2\left(f+\dot{W}_{1},\left(u_{t}+\delta u\right)\right) .
\end{aligned}
$$

Taking the real part of (37), we get

$$
\begin{gathered}
\frac{d}{d t}\left\|(-\Delta)^{\alpha / 2} u\right\|^{2}+2 \delta\left\|(-\Delta)^{\alpha / 2} u\right\|^{2}+2 \operatorname{Re} \int_{D} n u \bar{u}_{t} d x \\
+2 \delta \int_{D} n|u|^{2} d x+2 \operatorname{Re} \int_{D}\left(\bar{u}_{t}+\delta \bar{u}\right) \dot{W}_{1} d x \\
+2 \operatorname{Re} \int_{D} f \bar{u}_{t} d x+2 \delta \operatorname{Re} \int_{D} f \bar{u} d x=\left\|q_{1 x}\right\|^{2} .
\end{gathered}
$$

It is easy to check that

$$
\begin{aligned}
\frac{d}{d t} \int_{D} n|u|^{2} d x= & \int_{D} n_{t}|u|^{2} d x+2 \operatorname{Re} \int_{D} n u_{t} \bar{u} d x \\
& +2 \int_{D} q_{2} \operatorname{Im}\left(q_{1} \bar{u}\right) d x .
\end{aligned}
$$

Otherwise, by (2), we can have $n_{t}=-|u|_{x}^{2}-\beta n+g+\dot{W}_{2}$.

$$
\text { So }
$$

$$
\begin{aligned}
\int_{D} & n_{t}|u|^{2} d x=\int_{D}\left(-|u|_{x}^{2}-\beta n+g+\dot{W}_{2}\right)|u|^{2} d x \\
= & -\beta \int_{D} n|u|^{2} d x+\int_{D} g|u|^{2} d x+\int_{D} \dot{W}_{2}|u|^{2} d x \\
- & 2 \operatorname{Re} \int_{D}\left(\bar{u}_{t}+\delta \bar{u}\right) \dot{W}_{1} d x \\
= & 2 \operatorname{Im} \int_{D}\left(i \bar{u}_{t}+i \delta \bar{u}\right) \dot{W}_{1} d x \\
= & 2 \operatorname{Im} \int_{D}\left(\bar{f}+\dot{W}_{1}+(-\Delta)^{\alpha} \bar{u}+n \bar{u}\right) \dot{W}_{1} d x \\
= & 2 \operatorname{Im} \int_{D}(-\Delta)^{\alpha / 2} u(-\Delta)^{\alpha / 2} \dot{W}_{1} d x \\
& +2 \operatorname{Im} \int_{D}(n \bar{u}+\bar{f}) \dot{W}_{1} d x .
\end{aligned}
$$

From (2), we can obtain

$$
\begin{aligned}
& \frac{d}{d t}\left(\left\|(-\Delta)^{\alpha / 2} u\right\|^{2}+2 \operatorname{Re} \int_{D} f \bar{u} d x+\int_{D} n|u|^{2} d x\right) \\
& \quad+2 \delta\left\|(-\Delta)^{\alpha / 2} u\right\|^{2} \\
& =-2 \delta \int_{D} n|u|^{2} d x-2 \delta \operatorname{Re} \int_{D} f \bar{u} d x+\left\|q_{1 x}\right\|^{2} \\
& \quad+2 \operatorname{Im} \int_{D}(-\Delta)^{\alpha / 2} u(-\Delta)^{\alpha / 2} \dot{W}_{1} d x \\
& \quad+2 \operatorname{Im} \int_{D}(n \bar{u}+\bar{f}) \dot{W}_{1} d x+2 \int_{D} q_{2} \operatorname{Im}\left(q_{1} \bar{u}\right) d x \\
& \quad-\beta \int_{D} n|u|^{2} d x+\int_{D} g|u|^{2} d x+\int_{D} \dot{W}_{2}|u|^{2} d x .
\end{aligned}
$$

Using Hölder's inequality, the Gagliardo-Nirenberg inequality, and Young's inequality, we can estimate each term. Now, let $H_{0}=\left\|(-\Delta)^{\alpha / 2} u\right\|^{2}+2 \operatorname{Re} \int_{D} f \bar{u} d x+\int_{D} n|u|^{2} d x+\|n\|^{2}+$ 
$\int_{D} i\left(u_{x} \bar{u}-u \bar{u}_{x}\right) d x$ and take $\eta=\min \{\delta, \beta\}$. Putting (36) and

(41) into the result, we get

$$
\begin{aligned}
\frac{d}{d t} H_{0}+\eta H_{0} \leq & c\left(f, g, q_{1}, q_{2}\right)+c\|u\|^{6} \\
& +\int_{D}|u|^{2} \dot{W}_{2} d x+2 \int_{D} n \dot{W}_{2} d x \\
& -2 \operatorname{Im} \int_{D}(-\Delta)^{\alpha / 2} u(-\Delta)^{\alpha / 2} \dot{W}_{1} d x \\
& -2 \operatorname{Im} \int_{D}(n \bar{u}+\bar{f}) \dot{W}_{1} d x \\
& +4 \operatorname{Re} \int_{D} u \bar{W}_{1 x} d x .
\end{aligned}
$$

Multiplying by $e^{\eta t}$, integrating from 0 to $t$, and taking expectation on both sides of (42) yield

$$
\begin{gathered}
\mathbb{E} H_{0} \leq e^{-\eta t} \mathbb{E} H_{0}(0)+c\left(f, g, q_{1}, q_{2}\right) \\
+c \mathbb{E} \int_{0}^{t} e^{-\eta(t-s)}\|u\|^{6} d s .
\end{gathered}
$$

From (27), we can estimate (43) and obtain

$$
\begin{aligned}
\mathbb{E} H_{0}(t) & \leq e^{-\beta t} \mathbb{E} H_{0}(0)+c\left(f, g, q_{1}, q_{2}, u_{0}\right) \\
& \leq c\left(f, g, q_{1}, q_{2}, u_{0}\right) \leq C, \quad t>0,
\end{aligned}
$$

where $C$ is independent of $T$.

Since

$$
\begin{aligned}
H_{0}(t) \geq & \frac{1}{2}\left(\left\|(-\Delta)^{\alpha / 2} u\right\|^{2}+\|n\|^{2}\right) \\
& -c\left(\|u\|^{2}+\|g\|^{2}+\|u\|^{6}\right),
\end{aligned}
$$

for any $t>0$, we obtain

$$
\begin{aligned}
& \mathbb{E}\left(\left\|(-\Delta)^{\alpha / 2} u\right\|^{2}+\|n\|^{2}\right) \\
& \quad \leq c \mathbb{E}\left(\|u\|^{2}+\|g\|^{2}+\|u\|^{6}\right)+c \mathbb{E} H_{0}(t) \leq C,
\end{aligned}
$$

where $C$ is independent of $T$.

Besides, we estimate $H_{0}^{p}(t)$ for $p \geq 1$. Firstly, applying the Itô formula to $H_{0}^{p}(t)$, we have

$$
\begin{gathered}
\frac{d}{d t} H_{0}^{p}(t) \leq-\frac{\eta p}{2} H_{0}^{p}(t)+c\left(\|u\|^{6 p}+c\right)+p H_{0}^{p-1}(t) \\
\cdot\left(\int_{D}|u|^{2} \dot{W}_{2} d x+2 \int_{D} n \dot{W}_{2} d x\right)+p H_{0}^{p-1}(t) \\
\cdot\left(-2 \operatorname{Im} \int_{D}(-\Delta)^{\alpha / 2} u(-\Delta)^{\alpha / 2} \dot{W}_{1} d x\right. \\
\left.-2 \operatorname{Im} \int(n \bar{u}+\bar{f}) \dot{W}_{1} d x+4 \operatorname{Re} \int_{D} u \bar{W}_{1 x} d x\right) .
\end{gathered}
$$

Multiplying by $e^{(\eta p / 2) t}$, integrating from 0 to $t$, and taking expectation on both sides of (47) yield

$$
\begin{aligned}
\mathbb{E} H_{0}^{p}(t) \leq & e^{(-\eta p / 2) t} \mathbb{E} H_{0}^{p}(0)+c \\
& +c \mathbb{E} \int_{0}^{t} e^{(-\eta p / 2)(t-s)}\|u\|^{6 p} d s .
\end{aligned}
$$

From (27) and (48), we obtain

$$
\mathbb{E}\left(H_{0}^{p}(t)\right) \leq e^{(\eta p / 2) t} \mathbb{E} H_{0}^{p}(0)+c \leq C, \quad t>0,
$$

where $C$ is independent of $T$.

Consequently, from (45), we obtain

$$
\mathbb{E}\left(\left\|(-\Delta)^{\alpha / 2} u\right\|^{2}+\|n\|^{2}\right) \leq C, \quad t>0
$$

On the one side, integrating from 0 to $t$ on both sides of (42), we can deduce

$$
\begin{aligned}
& H_{0}(t) \leq H_{0}(0)+c\left(f, g, q_{1}, q_{2}\right) t+c \int_{0}^{t}\|u\|^{6} d s \\
& +\int_{0}^{t}\left(\int_{D}|u|^{2} \dot{W}_{2} d x+2 \int_{D} n \dot{W}_{2} d x\right) d s \\
& +\int_{0}^{t}\left(-2 \operatorname{Im} \int_{D}(-\Delta)^{\alpha / 2} u(-\Delta)^{\alpha / 2} \dot{W}_{1} d x\right. \\
& \quad-2 \operatorname{Im} \int_{D}(n \bar{u}+\bar{f}) \dot{W}_{1} d x \\
& \left.+4 \operatorname{Re} \int_{D} u \bar{W}_{1 x} d x\right) d s .
\end{aligned}
$$

Taking the supremum and expectation on both sides of (51) yields

$$
\begin{aligned}
& \mathbb{E} \sup _{0 \leq t \leq T} H_{0}(t) \leq \mathbb{E} H_{0}(0)+c\left(f, g, q_{1}, q_{2}\right) T+c \mathbb{E} \\
& \cdot \sup _{0 \leq t \leq T} \int_{0}^{t}\|u\|^{6} d s+\left.\left.\mathbb{E} \sup _{0 \leq t \leq T}\left|\int_{0}^{t} \int_{D}\right| u\right|^{2} \dot{W}_{2} d x d s\right|^{2} \\
& +\mathbb{E} \sup _{0 \leq t \leq T}\left|\int_{0}^{t} \int_{D} n \dot{W}_{2} d x d s\right|^{2}+\mathbb{E} \\
& \cdot \sup _{0 \leq t \leq T}\left|\int_{0}^{t} \operatorname{Im} \int_{D} \operatorname{Im} \int_{D}(-\Delta)^{\alpha / 2} u(-\Delta)^{\alpha / 2} \dot{W}_{1} d x d s\right|^{2} \\
& +\underset{0}{\operatorname{Esup}}\left|\int_{0 \leq t \leq T}^{t} \operatorname{Im} \int_{D}(n \bar{u}+\bar{f}) \dot{W}_{1} d x d s\right|^{2}+\mathbb{E} \\
& \cdot \sup _{0 \leq t \leq T}\left|\int_{0}^{t} \operatorname{Re} \int_{D} u \dot{W}_{1 x} d x d s\right|^{2} .
\end{aligned}
$$

And then by estimating each term of the right hand side of (52), we obtain

$$
\mathbb{E} \sup _{0 \leq t \leq T} H_{0}(t) \leq C_{T}\left(E_{0}, f, g, q_{1}, q_{2}\right) .
$$


On the other side, for $H_{0}^{p}(t)(p \geq 1)$, integrating from 0 to $t$ and taking the supremum and the expectation on both sides of (47) yield

$$
\begin{aligned}
& \mathbb{E} \sup _{0 \leq t \leq T} H_{0}^{p}(t) \leq \mathbb{E} H_{0}^{p}(0)+c\left(q_{1}, q_{2}\right) T+c \mathbb{E} \\
& \cdot \sup _{0 \leq t \leq T} \int_{0}^{t}\|u\|^{6 p} d s+c+p^{2} \mathbb{E} \sup _{0 \leq t \leq T} \mid \int_{0}^{t} H_{0}^{p-1}(s) \\
& \left.\cdot \int|u|^{2} \dot{W}_{2} d x d s\right|^{2}+p^{2} \mathbb{E} \sup _{0 \leq t \leq T} \mid \int_{0}^{t} 2 H_{0}^{p-1}(s) \\
& \left.\cdot \int n \dot{W}_{2} d x d s\right|^{2}+p^{2} \mathbb{E} \sup _{0 \leq t \leq T} \mid \int_{0}^{t} H_{0}^{p-1}(s) 4 \\
& \left.\cdot \operatorname{Re} \int_{D}(-\Delta)^{\alpha / 2} u(-\Delta)^{\alpha / 2} \dot{W}_{1} d x d s\right|^{2}+p^{2} \mathbb{E} \\
& \cdot \sup _{0 \leq t \leq T}\left|\int_{0}^{t} 2 H_{0}^{p-1}(s) \operatorname{Im} \int_{D} u \bar{W}_{1 x} d x d s\right|^{2}+p^{2} \mathbb{E} \\
& \cdot \sup _{0 \leq t \leq T}\left|\int_{0}^{t} 2 H_{0}^{p-1}(s) \operatorname{Im} \int(n \bar{u}+\bar{f}) \dot{W}_{1} d x d s\right|^{2} .
\end{aligned}
$$

Now we estimate each term of (54). For the third term on the right hand of (54),

$$
c \mathbb{E} \sup _{0 \leq t \leq T} \int_{0}^{t}\|u\|^{6 p} d s \leq c \mathbb{E} \int_{0}^{T}\|u\|^{6 p} d s \leq C_{T} .
$$

For the fifth and sixth terms on the right hand of (54), we have

$$
\begin{aligned}
& \left.\left.p^{2} \mathbb{E} \sup _{0 \leq t \leq T}\left|\int_{0}^{t} H_{0}^{p-1}(s) \int\right| u\right|^{2} \dot{W}_{2} d x d s\right|^{2}+p^{2} \mathbb{E} \\
& \cdot \sup _{0 \leq t \leq T}\left|\int_{0}^{t} 2 H_{0}^{p-1}(s) \int n \dot{W}_{2} d x d s\right|^{2} \leq c\left\|q_{2}\right\|^{2} \\
& \cdot \mathbb{E} \int_{0}^{T}\left(H_{0}^{2 p}(s)+\|u\|^{2 p}+\|u\|^{6 p}+\|f\|^{2 p}\right) d s \\
& \leq C_{T} .
\end{aligned}
$$

For the seventh and eight terms on the right hand of (54), using a similar method, we estimate

$$
\begin{aligned}
& p^{2} \mathbb{E} \sup _{0 \leq t \leq T} \mid \int_{0}^{t} H_{0}^{p-1}(s) 4 \\
& \left.\cdot \operatorname{Re} \int_{D}(-\Delta)^{\alpha / 2} u(-\Delta)^{\alpha / 2} \dot{W}_{1} d x d s\right|^{2}+p^{2} \mathbb{E} \\
& \cdot \sup _{0 \leq t \leq T}\left|\int_{0}^{t} 2 H_{0}^{p-1}(s) \operatorname{Im} \int_{D} u \bar{W}_{1 x} d x d s\right|^{2} \\
& \leq c\left\|(-\Delta)^{\alpha / 2} q_{1}\right\|^{2} \mathbb{E} \int_{0}^{T}\left(H_{0}^{2 p}(s)+\|u\|^{2 p}+\|u\|^{6 p}\right. \\
& \left.+\|f\|^{2 p}\right) d s \leq C_{T} .
\end{aligned}
$$

For the last term on the right hand of (54), we estimate

$$
\begin{aligned}
& p^{2} \mathbb{E} \sup _{0 \leq t \leq T}\left|\int_{0}^{t} 2 H_{0}^{p-1}(s) \operatorname{Im} \int(n \overline{\mathcal{u}}+\bar{f}) \dot{W}_{1} d x d s\right|^{2} \\
& \leq c\left\|(-\Delta)^{\alpha / 2} q_{1}\right\|^{2} \\
& \cdot \mathbb{E} \int_{0}^{T}\left(H_{0}^{2 p}(s)+\|u\|^{2 p}+\|u\|^{6 p}+\|f\|^{2 p}\right) d s \\
& \leq C_{T} .
\end{aligned}
$$

By the above estimates, we clearly obtain

$$
\begin{aligned}
\mathbb{E} \sup _{0 \leq t \leq T} H_{0}^{p}(t) & \leq \mathbb{E} H_{0}^{p}(0)+C_{T}\left(q_{1}, q_{2}, E_{0}, n_{1}, n_{0}\right) \\
& <C_{T}\left(q_{1}, q_{2}, E_{0}, n_{1}, n_{0}\right) .
\end{aligned}
$$

In addition, by (46), we can get

$$
\begin{aligned}
& \mathbb{E} \sup _{0 \leq t \leq T}\left(\left\|(-\Delta)^{\alpha / 2} u\right\|^{2 p}+\|n\|^{2 p}\right) \\
& \leq c \mathbb{E} \sup _{0 \leq t \leq T} H_{0}^{p}(t) \\
& \quad+c \mathbb{E} \sup _{0 \leq t \leq T}\left(\|u\|^{2 p}+\|u\|^{6 p}+\|f\|^{2 p}\right) \leq C_{T} .
\end{aligned}
$$

Then we obtain

$$
\mathbb{E} \sup _{0 \leq t \leq T}\|(u, n)\|_{V_{0}}^{2 p} \leq C_{T}\left(f, g, q_{1}, q_{2}, u_{0}, n_{0}\right) .
$$

Then the proof is complete.

\subsection{A Priori Estimates in $V_{1}$}

Lemma 5. Provided that $f, g \in H^{1}(D),\left(u_{0}, n_{0}\right) \in V_{1}, q_{1} \in$ $H^{2 \alpha}, q_{2} \in H^{1}(D)$, then for any $T>0$ and $p \geq 1$, we have $(n, u) \in L^{\infty}\left((0, \infty) ; L^{2 p}\left(\Omega ; V_{1}\right)\right) \cap L^{2 p}\left(\Omega ; L^{\infty}\left(0, T ; V_{1}\right)\right)$.

Proof. Taking the inner product of (1) with $(-\Delta)^{\alpha} u_{t}$, we get

$$
\begin{aligned}
& \left(i u_{t}-(-\Delta)^{\alpha} u-n u+i \delta u,(-\Delta)^{\alpha} u_{t}\right) \\
& =\left(f+\dot{W}_{1},(-\Delta)^{\alpha} u_{t}\right) .
\end{aligned}
$$

Applying the Itô formula to $\left\|(-\Delta)^{\alpha} u\right\|^{2}$, we have

$$
\begin{aligned}
\frac{d}{d t}\left\|(-\Delta)^{\alpha} u\right\|^{2}= & 2 \operatorname{Re} \int_{D}(-\Delta)^{\alpha} u(-\Delta)^{\alpha} \bar{u}_{t} d x \\
& +\left\|(-\Delta)^{\alpha} q_{1}\right\|^{2} .
\end{aligned}
$$

Since $u_{t}=i\left(-(-\Delta)^{\alpha} u-n u+i \delta u-f-\dot{W}_{1}\right)$ and

$$
\begin{aligned}
& \frac{d}{d t} \operatorname{Re} \int n u(-\Delta)^{\alpha} \bar{u} d x=\operatorname{Re} \int_{D}\left(n_{t} u(-\Delta)^{\alpha} \bar{u}\right. \\
& \quad+n u_{t}(-\Delta)^{\alpha} u+n u(-\Delta)^{\alpha} \bar{u}_{t}+q_{2}(-\Delta)^{\alpha} \bar{q}_{1} \\
& \left.\quad+i\left(q_{2}(-\Delta)^{\alpha} \bar{q}_{1} u-q_{2} q_{1}(-\Delta)^{\alpha} \bar{u}\right)\right) d x,
\end{aligned}
$$


we obtain

$$
\begin{aligned}
& \frac{d}{d t}\left(\left\|(-\Delta)^{\alpha} u\right\|^{2}\right. \\
& \left.-2 \operatorname{Re} \int_{D} n u(-\Delta)^{\alpha} \bar{u} d x+2 \operatorname{Re} \int_{D} f(-\Delta)^{\alpha} \bar{u} d x\right) \\
& +2 \alpha\left\|(-\Delta)^{\alpha} u\right\|^{2}=\left\|(-\Delta)^{\alpha} q_{1}\right\|^{2}+2\left\|q_{1 x}\right\|^{2}+2 \\
& \cdot \operatorname{Re} \int_{D} i\left(q_{2} q_{1}(-\Delta)^{\alpha} \bar{u}-q_{2}(-\Delta)^{\alpha} \bar{q}_{1} u\right) d x-2 \\
& \cdot \operatorname{Re} \int_{D} n u_{t}(-\Delta)^{\alpha} u d x+2 \delta \operatorname{Re} \int_{D} n u(-\Delta)^{\alpha} \bar{u} d x \\
& -2 \delta \operatorname{Re} \int_{D} f(-\Delta)^{\alpha} u d x-2 \\
& \quad \operatorname{Re} \int_{D} \dot{W}_{1}\left((-\Delta)^{\alpha} \bar{u}_{t}+\delta(-\Delta)^{\alpha} \bar{u}\right) d x-2 \\
& \cdot \operatorname{Re} \int_{D} n_{t} u(-\Delta)^{\alpha} \bar{u} d x .
\end{aligned}
$$

According to the above inequality, we can estimate each term on the right hand side of (65) using Höder's inequality, the Gagliardo-Nirenberg, and Young's inequality. So, by (65), we get

$$
\begin{aligned}
& \frac{d}{d t}\left(\left\|(-\Delta)^{\alpha} u\right\|^{2}-2 \operatorname{Re} \int_{D} n u(-\Delta)^{\alpha} \bar{u} d x+2\right. \\
& \left.\quad \cdot \operatorname{Re} \int_{D} f(-\Delta)^{\alpha} \bar{u} d x\right)+2 \alpha\left\|(-\Delta)^{\alpha} u\right\|^{2} \\
& \quad \leq \frac{\delta}{2}\left\|(-\Delta)^{\alpha} u\right\|^{2}+\frac{\beta}{2}\left\|n_{x}\right\|^{2}+c\left(\left\|q_{2}\right\|_{H_{1}}^{4}+\left\|q_{1}\right\|_{H^{2 \alpha}}^{2}\right. \\
& \left.\quad+\|g\|^{4}+\|f\|^{8}+\|u\|_{H_{1}}^{8}+\|n\|^{12}\right)-2 \\
& \quad \cdot \operatorname{Re} \int_{D} \dot{W}_{1}(-\Delta)^{\alpha} \bar{u}_{t} y-2 \operatorname{Re} \int_{D} u(-\Delta)^{\alpha} \bar{u} \dot{W}_{2} d x \\
& \quad+2 \operatorname{Re} \int_{D} i n(-\Delta)^{\alpha} \bar{u}_{1} d x .
\end{aligned}
$$

Otherwise, applying the Itô formula to $\left\|n_{x}\right\|^{2}$, we have

$$
\frac{d}{d t}\left\|n_{x}\right\|^{2}=2 \int_{D} n_{x} n_{x t} d x+\left\|q_{2 x}\right\|^{2}
$$

Since $n_{t}=g+\dot{W}_{2}-\beta n-|u|_{x}^{2}$ and

$$
\begin{aligned}
& \frac{d}{d t} i \int_{D}\left(u_{x}(-\Delta)^{\alpha} \bar{u}-(-\Delta)^{\alpha} u \bar{u}_{x}\right) d x \\
& =2 i \int_{D}\left(u_{x t}(-\Delta)^{\alpha} \bar{u}-\bar{u}_{x t}(-\Delta)^{\alpha} u\right) d x \\
& \quad+2 i \operatorname{Re} \int_{D} q_{1 x}(-\Delta)^{\alpha} \bar{q}_{2} d x
\end{aligned}
$$

we obtain

$$
\begin{aligned}
& \frac{d}{d t}\left(\left\|n_{x}\right\|^{2}+i \int_{D}\left(u_{x}(-\Delta)^{\alpha} \bar{u}-(-\Delta)^{\alpha} u \bar{u}_{x}\right) d x\right) \\
& \quad+2 \beta\left\|n_{x}\right\|^{2} \\
& \leq \frac{\delta}{2}\left\|(-\Delta)^{\alpha} u\right\|^{2}+\frac{\beta}{2}\left\|n_{x}\right\|^{2} \\
& \quad+c\left(\left\|g_{x}\right\|^{2}+\left\|f_{x}\right\|^{2}+\left\|q_{1}\right\|_{H^{2 \alpha}}^{2}+\left\|q_{2 x}\right\|^{2}\right) \\
& \quad+2 \int_{D} n_{x} \dot{W}_{2 x} d x+4 \operatorname{Re} \int_{D}(-\Delta)^{\alpha} \bar{u} \dot{W}_{1 x} d x .
\end{aligned}
$$

Thus, by (66) and (69), taking $\eta=\min \{\delta, \beta\}$, and letting

$$
\begin{aligned}
H_{1}(t)= & \left\|(-\Delta)^{\alpha} u\right\|^{2}+\left\|n_{x}\right\|^{2}-2 \operatorname{Re} \int_{D} n u(-\Delta)^{\alpha} \bar{u} d x \\
& +2 \operatorname{Re} \int_{D} f(-\Delta)^{\alpha} \bar{u} d x \\
& +i \int_{D}\left(u_{x}(-\Delta)^{\alpha} \bar{u}-(-\Delta)^{\alpha} u \bar{u}_{x}\right) d x
\end{aligned}
$$

we get

$$
\begin{aligned}
& \frac{d}{d t} H_{1}(t)+\eta H_{1}(t) \leq c\left(\|u\|_{H^{1}}^{8}+\|n\|^{12}+\left\|q_{2}\right\|_{H_{1}}^{4}\right. \\
& \left.+\left\|q_{1}\right\|_{H^{2 \alpha}}^{4}+\|g\|_{H^{1}}^{4}+\|f\|_{H^{1}}^{8}+1\right)-2 \\
& \cdot \operatorname{Re} \int_{D} \dot{W}_{1}(-\Delta)^{\alpha} \bar{u}_{t} y-2 \operatorname{Re} \int_{D} u(-\Delta)^{\alpha} \bar{u} \dot{W}_{2} d x \\
& +2 \operatorname{Re} \int_{D} i n(-\Delta)^{\alpha} \bar{u} \dot{W}_{1} d x+2 \int_{D} n_{x} \dot{W}_{2 x} d x+4 \\
& \quad \cdot \operatorname{Re} \int_{D}(-\Delta)^{\alpha} \bar{u} \dot{W}_{1 x} d x .
\end{aligned}
$$

Multiplying by $e^{\eta t}$, integrating from 0 to $t$, and taking expecting on both sides of (71), by (46), we obtain

$$
\begin{aligned}
\mathbb{E} H_{1}(t) & \leq e^{-\beta t} \mathbb{E} H_{1}(0)+c\left(f, g, q_{1}, q_{2}, u_{0}\right) \\
& \leq c\left(f, g, q_{1}, q_{2}, u_{0}\right) \leq C, \quad t>0,
\end{aligned}
$$

where $C$ is independent of $T$. Since

$$
\begin{aligned}
H_{1}(t) \geq & \frac{1}{2}\left(\left\|(-\Delta)^{\alpha} u\right\|^{2}+\left\|n_{x}\right\|^{2}\right) \\
& -c\left(\left\|u_{x}\right\|^{8}+\|n\|^{4}+1+\|f\|^{2}\right),
\end{aligned}
$$

for any $t>0$, we obtain

$$
\begin{aligned}
& \mathbb{E}\left(\left\|(-\Delta)^{\alpha} u\right\|^{2}+\left\|n_{x}\right\|^{2}\right) \\
& \quad \leq c \mathbb{E}\left(\left\|u_{x}\right\|^{8}+\|n\|^{4}+1+\|f\|^{2}\right)+c \mathbb{E} H_{1}(t) \leq C .
\end{aligned}
$$

where $C$ is independent of $T$. 
Next step, we estimate $H_{1}^{p}(t)$ for $p \geq 1$. On the one hand, applying the Itô formula to $H_{1}^{p}(t)$, we obtain

$$
\begin{aligned}
& \mathbb{E} H_{1}^{p}(t) \\
& \leq e^{(-\eta t / 2) t} \mathbb{E} H_{1}^{p}(0)+c \\
& \quad+c \mathbb{E} \int_{0}^{t} e^{(-\eta t / 2)(t-s)}\left(\left\|(-\Delta)^{\alpha} u\right\|^{8 p}+\|n\|^{12 p}+1\right) d s .
\end{aligned}
$$

By (45), we obtain

$$
\mathbb{E} H_{0}^{p}(t) \leq e^{-(\eta p / 2) t} \mathbb{E} H_{0}^{p}(0)+c \leq C, \quad t>0,
$$

where $C$ is independent of $T$. Thus, from (73), for any $t>0$, we get

$$
\mathbb{E}\left(\left\|(-\Delta)^{\alpha} u\right\|^{2 p}+\left\|n_{x}\right\|^{2 p}\right) \leq C .
$$

Integrating from 0 to $t$ and taking the supremum and expectation on both sides of (71), as with the estimates in Lemma 4 for each term, we deduce

$$
\mathbb{E} \sup _{0 \leq t \leq T} H_{1}(t) \leq C_{T}\left(f, g, q_{1}, q_{2}, u_{0}\right) .
$$

On the other hand, for $H_{0}^{p}(t)(p \geq 1)$, integrating from 0 to $t$ and taking the supremum and the expectation on both sides of (71), and estimating each term, we get

$$
\begin{aligned}
\mathbb{E} \sup _{0 \leq t \leq T} H_{1}^{p}(t) & \leq \mathbb{E} H_{1}^{p}(0)+C_{T}\left(f, g, q_{1}, q_{2}, u_{0}, n_{0}\right) \\
& \leq C_{T}\left(f, g, q_{1}, q_{2}, u_{0}, n_{0}\right) .
\end{aligned}
$$

Therefore, from (73), it is inferred that for $p \geq 1$

$$
\begin{gathered}
\mathbb{E} \sup _{0 \leq t \leq T}\left(\left\|(-\Delta)^{\alpha} u\right\|^{2 p}+\left\|n_{x}\right\|^{2 p}\right) \\
\leq C_{T}\left(f, g, q_{1}, q_{2}, u_{0}, n_{0}\right) .
\end{gathered}
$$

So then, we have

$$
\mathbb{E} \sup _{0 \leq t \leq T}\|(u, n)\|_{V_{1}}^{2 p} \leq C_{T}\left(f, g, q_{1}, q_{2}, u_{0}, n_{0}\right),
$$

and the proof is complete.

3.3. A Priori Estimates in $V_{2}$. By using the similar method and idea as Sections 3.1 and 3.2, we can achieve a priori estimates in $V_{2}$. For simplicity, we only provide the idea of the proof. Using (1)-(2) and applying the Itô formula to $\left\|(-\Delta)^{\alpha} n\right\|^{2}$ and $\left\|(-\Delta)^{\alpha} u_{x}\right\|^{2}$, respectively, we can get some inequalities by Höder's inequality, the Gagliardo-Nirenberg, and Young's inequality. After that, taking the supremum and expectation for inequalities and estimating on $\left\|(-\Delta)^{\alpha} n\right\|^{2}$ and $\left\|(-\Delta)^{\alpha} u_{x}\right\|^{2}$ by Gronwall-type, we can deduce the following lemma.

Lemma 6. Provided that $\left(u_{0}, n_{0}\right) \in V_{2}, q_{2} \in H^{2}(D), q_{1} \in$ $H^{3 \alpha}(D)$, and $f, g \in H^{2}(D)$, then for any $T>0$ and $p \geq 1$, we have $(u, n) \in L^{\infty}\left(0, \infty ; L^{2 p}\left(\Omega ; V_{2}\right)\right) \cap L^{2 p}\left(\Omega ; L^{\infty}\left(0, T ; V_{2}\right)\right)$.

\section{Proofs of Theorems 7 and 8}

Based on the prior estimate, we acquire the existence and uniqueness of a solution for the stochastic fractional longshort wave equations (1)-(2) in spaces $V_{1}$.

Theorem 7. If $\left(u_{0}, n_{0}\right) \in V_{1}, q_{2} \in H^{1}(D), q_{1} \in H^{2 \alpha}(D)$, and $f, g \in H^{1}(D)$, then there exists a unique solution $(u, n) \in$ $\left(\mathbb{R}^{+} ; V_{1}\right)$, almost surely satisfying (1)-(2). In addition, $(u, n)$ is continuous from $\mathbb{R}^{+}$to $V_{1}$.

Proof. First, we know that $\left(n_{0}, u_{0}\right) \in V_{1}$. Let $\left\{e_{i}(x)\right\}_{i=1}^{\infty}$ be an orthonormal basis of eigenvectors of the Laplace operator on $D$, which is an orthonormal basis of $L^{2}(D)$. Consider $P^{k}$ as the projection from $L^{2}(D)$ onto the space spanned by $\left\{e_{i}: i=\right.$ $1,2, \ldots, k\}$. Then the approximation solution $\left(u^{k}, n^{k}\right)$ solves the approximation problem

$$
\begin{array}{r}
i u_{t}^{k}-(-\Delta)^{\alpha} u^{k}-P^{k}\left(n^{k} u^{k}\right)+i \delta u^{k}=\dot{W}_{1}^{k}+f^{k}, \\
n_{t}^{k}+P^{k}\left(\left|u^{k}\right|^{2}\right)_{x}+\beta n^{k}=g^{k}+\dot{W}_{2}^{k},
\end{array}
$$

where $P^{k}$ is the projector onto the first $k$ vectors $e_{i}, \dot{W}_{1}^{k}=$ $P^{k} \dot{W}_{1}, \dot{W}_{2}^{k}=P^{k} \dot{W}_{2}$, and $P^{k}$ commutes with the operator $(-\Delta)^{\alpha}$. We will treat the above equations pathwise by introducing the following random processes solving

$$
\begin{aligned}
i \xi_{t}^{k}-(-\Delta)^{\alpha} \xi^{k}+i \delta \xi^{k} & =\dot{W}_{1}^{k}, \\
\zeta^{k}+\beta \zeta^{k} & =\dot{W}_{2}^{k},
\end{aligned}
$$

with periodic boundary conditions and initial conditions

$$
\begin{aligned}
\zeta(x, t) & =\eta(x+2 \pi, t), \\
\xi(x, t) & =\xi(x+2 \pi, t)=0, \\
\zeta_{t}(x, 0) & =0, \\
\zeta(x, 0) & =0, \\
\xi(0, t) & =0, \\
x & \in D,
\end{aligned}
$$

where $\delta>0, \beta>0$.

In accordance with the same method as in Section 3, for any $T>0$ and almost all $\omega \in \Omega$, we have

$$
\begin{aligned}
& \zeta \in C\left(0, T ; H_{0}^{1}(D)\right), \\
& \xi \in C\left(0, T ; H^{2 \alpha}(D) \cap H_{0}^{\alpha}(D)\right) .
\end{aligned}
$$

Therefore, we can get the following estimate:

$$
\mathbb{E}\left(\left\|\eta_{x}\right\|^{2}+\left\|(-\Delta)^{\alpha} \xi\right\|^{2}\right) \leq C,
$$

where a positive constant $C$ is independent of $T$. Moreover, for any $T>0$,

$$
\mathbb{E} \sup _{0 \leq t \leq T}\left(\left\|\eta_{x}\right\|^{2}+\left\|(-\Delta)^{\alpha} \xi\right\|^{2}\right) \leq C_{T}
$$


holds for a positive constant $C_{T}$ dependent on $T$. Set $B^{k, M}=$ $\left(U^{k, M}, N^{k, M}\right)$ as the solution of the following equations:

$$
\begin{aligned}
& i U_{t}^{k, M}-(-\Delta)^{\alpha} U^{k, M}-\chi_{M}\left(\left\|B^{k, M}\right\|_{V_{1}}\right) P^{k}\left(n^{k, M} u^{k, M}\right) \\
& \quad+i \delta U^{k, M}=f^{k}, \\
& N_{t}^{k, M}+\chi_{M}\left(\left\|B^{k, M}\right\|_{V_{1}}\right) P^{k}\left(\left|u^{k, M}\right|^{2}\right)_{x}+\beta N^{k, M}=g^{k}, \\
& N^{k, M}(x, 0)=P^{k} n_{0}, \\
& U^{k, M}(x, 0)=P^{k} u_{0} .
\end{aligned}
$$

Let $n^{k, M}=N^{k, M}+P^{k} \zeta, u^{k, M}=U^{k, M}+P^{k} \xi$, and $\chi_{M} \in C_{0}^{\infty}(\mathbb{R})$ satisfy $\chi_{M}(r)=1$ for $|r| \leq M$ and $\chi_{M}(r)=0$ for $|r| \geq 2 M$. It is easy to see that (88)-(89) are random differential equations with Lipschitz nonlinearity in finite dimension. Afterwards, for almost all $\omega \in \Omega$, we have a unique solution $\left(U^{k, M}, N^{k, M}\right)$ for (88)-(89). We define the stopping time as follows:

$$
\tau_{M}=\inf \left\{t>0:\|B\|_{V_{1}}^{k, M} \geq M\right\},
$$

if the set $\left\{\|B\|_{M}^{k, M} \geq M\right\}$ is nonempty, or else $\tau_{M}=\infty$. Because $\tau_{M}$ is increasing in $M$, let $\tau_{\infty}=\lim _{M \rightarrow \infty} \tau_{M}$ almost surely. For $t<\tau_{M}$, we obtain

$$
\left(U^{k, M}, N^{k, M}\right)+\left(P^{k} \xi, P^{k} \zeta\right)
$$

satisfying (82). On the basis of the estimates given in Section 3.2 and (86)-(87), for any $t \geq 0$, we obtain

$$
\mathbb{E}\left\|\left(U^{k, M}, N^{k, M}\right)\right\|_{V_{1}}^{2} \leq C\left(f, g, q_{1}, q_{2}, n_{0}, u_{0}\right),
$$

where the positive constant $C\left(f, g, q_{1}, q_{2}, n_{0}, u_{0}\right)$, which is dependent on $T$ and $M$. And for $T>0$, we obtain

$$
\begin{array}{r}
\mathbb{E} \sup _{0 \leq t \leq T \wedge \tau_{M}}\left\|\left(U^{k, M}, N^{k, M}\right)\right\|_{V_{1}}^{2} \\
\quad \leq C_{T}\left(f, g, q_{1}, q_{2}, n_{0}, u_{0}\right),
\end{array}
$$

with the positive constant $C\left(f, g, q_{1}, q_{2}, n_{0}, u_{0}\right)$, which is dependent on $T$ but independent of $M$. Let $T \wedge \tau_{M}=$ $\min \left\{T, \tau_{M}\right\}$. On the other side, we get

$$
\begin{aligned}
& \mathbb{E}\left\|U^{k, M}\left(T \wedge \tau_{M}\right), N^{k, M}\left(T \wedge \tau_{M}\right)\right\|_{V_{1}}^{2} \\
& \quad \geq \mathbb{E}\left[I\left(\tau_{M} \leq T\right)\right. \\
& \left.\cdot\left|\left(U^{k, M}\left(T \wedge \tau_{M}\right), N^{k, M}\left(T \wedge \tau_{M}\right)\right)\right|_{V_{1}}^{2}\right] \geq M^{2} \mathbb{P}(\tau \\
& \quad \leq T),
\end{aligned}
$$

where $I\left(\tau_{M} \leq T\right)=1$ for $\tau_{M} \leq T$ and $I\left(\tau_{M} \leq T\right)=0$ for $\tau_{M}>T$. Then, by (93), we get

$$
\mathbb{P}\left(\tau_{M} \leq T\right) \leq \frac{1}{M^{2}} C\left(f, g, q_{1}, q_{2}, n_{0}, u_{0}\right) .
$$

In the light of the above estimate and the Borel-Cantelli lemma, for any $T>0$, we obtain

$$
\mathbb{P}\left(\tau_{\infty}>T\right)=1 .
$$

So we know that

$$
\left(U^{k}, N^{k}\right)=\lim _{M \rightarrow \infty}\left(U^{k, M}, N^{k, M}\right)
$$

satisfies the following random differential equations:

$$
\begin{array}{r}
i U_{t}^{k}-(-\Delta)^{\alpha} U^{k}-P^{k}\left(n^{k}, u^{k}\right)+i \delta U^{k}=f^{k}, \\
N_{t}^{k}+P^{k}\left(\left|u^{k, M}\right|^{2}\right)_{x}+\beta N^{k}=g^{k},
\end{array}
$$

with initial conditions

$$
\begin{aligned}
& U^{k}(0)=P^{k} u_{0}, \\
& N^{k}(0)=P^{k} n_{0} .
\end{aligned}
$$

Then $\left(U^{k}, N^{k}\right)$ satisfies the estimates (93) and (94), and for any $t \geq 0$, we find that $\left(u^{k}, n^{k}\right)=\left(U^{k}, N^{k}\right)+\left(P^{k} \xi, P^{k} \zeta\right)$ is the unique global solution of (82).

And then, we will investigate (99)-(100) for fixed $\omega$. Firstly, by (94), for any $T>0$, we can know that

$$
\mathbb{P}\left(\bigcup_{L=1}^{\infty} \bigcap_{l=1}^{\infty} \cap_{k=l}^{\infty}\left\{\sup _{0 \leq t \leq T}\left\|\left(U^{k}, N^{k}\right)\right\|_{V_{1}}^{2} \geq L\right\}\right)=0 .
$$

We let

$$
\widetilde{\Omega}=\bigcup_{L=1}^{\infty} \bigcap_{l=1}^{\infty} \cap_{k=l}^{\infty}\left\{\sup _{0 \leq t \leq T}\left\|\left(U^{k}, N^{k}\right)\right\|_{V_{1}}^{2} \leq L\right\} .
$$

Then $\mathbb{P}(\Omega \backslash \widetilde{\Omega})=0$. Thus, for any fixed $\omega \in \widetilde{\Omega}$, there exists $r(\omega)$ with $0<r(\omega)<\infty$ such that

$$
\sup _{0 \leq t \leq T}\left\|\left(U^{k}, N^{k}\right)\right\|_{V_{1}}^{2} \leq r(\omega) .
$$

Then we can extract a subsequence of $\left(U^{k}, N^{k}\right)$, which are still denoted by $\left(U^{k}, N^{k}\right)$, such that

$$
\begin{aligned}
& U^{k} \stackrel{*}{\rightarrow} U \quad \text { in } L^{\infty}\left(0, T ; H^{2 \alpha}(D) \cap H_{0}^{\alpha}(D)\right), \\
& N^{k *} \rightarrow N \quad \text { in } L^{\infty}\left(0, T ; H_{0}^{1}(D)\right) .
\end{aligned}
$$

These convergences are sufficient to pass the limit $k \rightarrow \infty$ in linear terms; however, in fact, for nonlinear terms, we need a strong convergence of $U^{k}$. From (100) and the estimate (104), it is easy to get $U_{t}^{k} \in L^{\infty}\left(0, T ; L^{2}(D)\right)$. Further, we can extract a subsequence of $U^{k}$ which is still denoted by $U^{k}$ such that

$$
U^{k} \longrightarrow U \text { strongly in } L^{\infty}\left(0, T ; H_{0}^{1}(D)\right) .
$$

For the nonlinear term, we can pass the limit $k \rightarrow \infty$ by a standard procedure. Thus, we prove that $(U, N) \in$ $L^{\infty}\left(0, T ; V_{1}\right)$ is a weak solution of

$$
\begin{array}{r}
i U_{t}-(-\Delta)^{\alpha} U-n u+i \delta U=f, \\
N_{t}+\left(|u|^{2}\right)_{x}+\beta N=g
\end{array}
$$


with initial conditions

$$
\begin{aligned}
& U(0)=u_{0}, \\
& N(0)=n_{0}, \\
& x \in D .
\end{aligned}
$$

Then $(u, n)=(U, N)+(\xi, \zeta)$ is a solution of $(1)-(2)$ and satisfies the estimates given in Section 3.2. The continuity of the solution can be proved in the following. For any $\omega \in \widetilde{\Omega},\left(|u|^{2}\right)_{x} \in L^{\infty}\left(0, T ; H_{1}^{0}(D)\right)$, it is obvious that $N_{t}=$ $-\left(|u|^{2}\right)_{x}-\beta N+g \in H_{0}^{1}(D)$. Then, we obtain that there is $N \in C\left(0, T ; H_{0}^{1}(D)\right)$ for almost all $\omega \in \Omega$ by Lemma 3 in [23]. Noticing $U_{t} \in L^{\infty}\left(0, T ; L^{2}(D)\right)$ almost surely and applying similar methods, we can get $U \in C\left(0, T ; H^{2 \alpha}(D) \cap H_{0}^{\alpha}(D)\right)$ according to [21]. So we get $(u, n) \in C\left(0, T ; V_{1}\right)$ almost surely by definition of $U$ and $N$. Thus, the solution $(u, n)$ is continuous from $[0, T]$ to $V_{1}$ almost surely.

Because the noise is additive, we can use the same approach as [7]. Therefore, the solution $(u, n)$ is unique almost surely in $L^{\infty}\left(0, T ; V_{1}\right)$.

Theorem 8. If $\left(u_{0}, n_{0}\right) \in V_{2}, q_{2} \in H^{2}(D), q_{1} \in H^{3 \alpha}(D)$, and $f, g \in H^{2}(D)$, then there exists a unique solution $(u, n) \in\left(\mathbb{R}^{+} ; V_{2}\right)$ almost surely satisfy (1)-(2). Moreover, $(u, n)$ is continuous from $\mathbb{R}^{+}$to $V_{2}$.

As a matter of fact, from Theorems 7 and 8 , a continuous random dynamical system can be defined in $V_{1}$ and $V_{2}$, respectively. Then we can, respectively, construct a random attractor endowed with the weak topology for the continuous random dynamical system in $V_{1}$ and $V_{2}$.

The proof of Theorem 8 is similar to that of Theorem 7 . Here we omit the detail of the proof.

\section{Proofs of Theorems 10 and 11}

In this section, the asymptotic behavior of solution for the corresponding problem is studied. We will construct a random attractor for stochastic fractional long-short wave equations in $v_{1}$ equipped with the weak topology. In [1316], we can obtain some basic concepts related to random attractors for random dynamical systems.

Motivated by $[13,14]$, we can find the following existence result for a random attractor for a continuous RDS. It is a sufficient condition for the existence of random attractors.

Theorem 9 (see $[13,14])$. Assume $\Phi$ is a RDS on a Polish space $(E, d)$ and there exists a random compact set $K(\omega)$ absorbing every bounded deterministic set $D \subset E$. Then we define a global random attractor for $R D S \Phi$ by

$$
\mathscr{A}(\omega)=\bigcap_{\tau \geq 0} \overline{\bigcup_{t \geq \tau} \Phi\left(t, \theta_{-t} \omega, K\left(\theta_{-t} \omega\right)\right)} .
$$

Next step, according to Theorem 9 and the priori estimates in Section 3, we research the random attractors for the stochastic long-short wave equations in $V_{1}$ and $V_{2}$.
Theorem 10. If $\left(u_{0}, n_{0}\right) \in V_{1}, q_{1} \in H^{1}(D), q_{2} \in H^{2 \alpha}(D)$, and $f, g \in H^{1}(D)$, then (1)-(2) have a global random weak attractor $\mathscr{A}(\omega)$ which is a random tempered compact set in $V_{1}$ endowed with the weak topology.

Proof. On the basis of the former analysis, we can consider the properties of solution a $(U, N)$ of the system and it (107) has a unique solution $(U, N) \in C\left(0, T ; V_{1}\right)$ for almost all $\omega \in \Omega$. Noticing that system (107) has coefficients driven by $\theta_{t}$, a random dynamical system can be defined by $(U, N)$ on $V_{1}$. Thus, $(u, n)=(U+\xi, N+\zeta)$ also defines a continuous random dynamical system on $V_{1}$, which is denoted by $\Phi(t, \omega)$, and $\Phi(t, \omega)$ is weakly continuous almost surely on $V_{1} . B(0, r)$ denotes the ball center at 0 with radius $r$ in $V_{1}$. Using estimates made in accordance with Section 3 , there is a random variable $R(\omega)$ such that, for any $r>0,(u, n) \in B(0, r)$. Therefore, there exists a random time $t_{r}(\omega)>0$, such that, for all $t>t_{r}(\omega)$ and almost all $\omega \in \Omega$,

$$
\left\|\Phi\left(t, \theta_{-t} \omega\right)\left(u_{0}, n_{0}\right)\right\|_{V_{1}} \leq R(\omega) .
$$

We define the random attractor $\mathscr{A}(\omega)$ as follows:

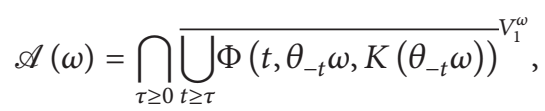

where the closure is taken with respect to the weak topology of $V_{1}$. Next we prove that $\mathscr{A}(\omega)$ is tempered. According to the estimates obtained in Section 3, we obtain

$$
\mathbb{E} \sup _{0 \leq t \leq 1} R^{2}\left(\theta_{t} \omega\right)<\infty .
$$

Then, from Birkhoff's ergodic Theorem [24],

$$
\lim _{s \rightarrow \pm \infty} \frac{\sup _{t \in[0,1]} R^{2}\left(\theta_{t+s} \omega\right)}{s}=0
$$

on a $\theta$-invariant subset of $\Omega$ with full probability measure; that is, $R(\omega)$ is tempered. So we get that $\mathscr{A}(\omega)$ is tempered. The proof is completed.

Theorem 11. If $\left(u_{0}, n_{0}\right) \in V_{2}, q_{1} \in H^{2}(D), q_{2} \in H^{3 \alpha}(D)$, and $f, g \in H^{2}(D)$, then (1)-(2) have a global random weak attractor $\mathscr{A}(\omega)$ which is a random tempered compact set in $V_{2}$ endowed with the weak topology.

Based on Theorem 10, we can prove Theorem 11 using the same methods and ideas of proof. More precisely, there exists a random attractor for the stochastic fractional longshort wave equations in $V_{2}$.

Theorem 12. If $\left(u_{0}, n_{0}\right) \in V_{2}, q_{1} \in H^{2}(D), q_{2} \in H^{4 \alpha}(D)$, then (1)-(2) have one stationary measure on $V_{1}$ and $V_{2}$.

Proof. If $\left(u_{0}, n_{0}\right) \in V_{2},(1)-(2)$ has a unique solution $(u, n)$ with $(u(0), n(0))=\left(u_{0}, n_{0}\right)$ by the results given in Sections 3 and 4 , which, for any $t>0$, satisfies

$$
\mathbb{E}\left(\left\|(-\Delta)^{\alpha} n\right\|^{2}+\left\|(-\Delta)^{\alpha} u_{x}\right\|^{2}\right) \leq C,
$$

for a positive constant $C>0$ which is independent of $t>0$. 
Let $\mu_{t}$ be the distribution of $\left(n_{t}, n, E\right)$ for $t \geq 0$. According to the classical Bogolyubov-Krylov argument [22], we define

$$
\bar{\mu}_{t}=\frac{1}{t} \int_{0}^{t} \mu_{s} d s
$$

as

$$
\bar{\mu}_{t}(\Gamma)=\frac{1}{t} \int_{0}^{t} \mu_{s}(\Gamma) d s
$$

for any Borel set $\Gamma$ of $V_{1}$ : namely, $\Gamma \in \mathscr{B}\left(V_{1}\right)$. From (114), we obtain

$$
\begin{aligned}
\int_{V_{1}}\|(u, n)\|_{V_{2}}^{2} \bar{\mu}_{t}(d v) & =\frac{1}{t} \int_{0}^{t} \mathbb{E}\|(u(s), n(s))\|_{V_{2}}^{2} d s \\
& \leq C .
\end{aligned}
$$

According to Chebyshev's inequality and the fact that $V_{2}$ has a compact embedding into $V_{1},\left\{\bar{\mu}_{t}\right\}_{t \geq 0}$ is tight in $V_{1}$. Then there exists a sequence $\left\{\bar{\mu}_{t k}\right\}$ with $t_{k} \rightarrow \infty$ as $k \rightarrow \infty$ and a probability measure $\mu$ on $V_{1}$ such that $\left\{\bar{\mu}_{t k}\right\} \rightarrow \mu$ weakly as $k \rightarrow \infty$. Thus, using the standard argument as in [24], $\mu$ is a stationary measure for stochastic fractional long-short wave equations on $V_{1}$. In addition, from (114), $\mu$ is in fact supported on $V_{2}$; that is to say, $\mu$ is a stationary measure for stochastic fractional long-short wave equations on $V_{2}$. The proof is completed.

\section{Conflicts of Interest}

The authors declare that there are no conflicts of interest regarding the publication of this article.

\section{Acknowledgments}

This work was supported by the This work was supported by the NSF of China (no. 11371183) and the NSF of Shandong Province (no. ZR2013AM004).

\section{References}

[1] R. H. J. Grimshaw, "Modulation of an internal gravity-wave packet, and the resonance with the mean motion," Studies in Applied Mathematics, vol. 56, no. 3, pp. 241-266, 1977.

[2] D. R. Nicholson and M. V. Goldman, "Damped nonlinear Schrödinger equation," The Physics of Fluids, vol. 19, no. 10, pp. 1621-1625, 1976.

[3] B. Guo, "The global solution for one class of the system of LS nonlinear wave interaction," Journal of Mathematical Research and Exposition, vol. 7, no. 1, pp. 69-76, 1987.

[4] B. L. Guo, "The periodic initial value problems and initial value problems for class of generalized long-short type equations," $J$. Engineering Math, vol. 8, pp. 47-53, 1991.

[5] X. Y. Du and B. L. Guo, "The global attracttor for LS type equation in R1," Acta Mathematicae Applicatae Sinica, vol. 28, pp. 723-734, 2005.

[6] B. Guo and L. Chen, "Orbital stability of solitary waves of the long wave-short wave resonance equations," Mathematical Methods in the Applied Sciences, vol. 21, no. 10, pp. 883-894, 1998.
[7] B. Guo and B. Wang, "Attractors for the long-short wave equations," Journal of Partial Differential Equations, vol. 11, no. 4, pp. 361-383, 1998.

[8] Y. Li, "Long time behavior for the weakly damped driven longwave-short-wave resonance equations," Journal of Differential Equations, vol. 223, no. 2, pp. 261-289, 2006.

[9] R.-F. Zhang, "Existence of global attractor for LS type equations," Journal of Mathematical Research and Exposition, vol. 26, no. 4, pp. 708-714, 2006.

[10] E. Weinan, X. Li, and E. Vanden-Eijnden, "Some recent progress in multiscale modeling," in Multiscale Modelling and Simulation, P. Koumoutsakos and S. Attiner, Eds., vol. 39 of Lecture Notes in Computational Science and Engineering, pp. 3-21, Springer, Berlin, Germany, 2004.

[11] P. Imkeller and A. H. Monahan, "Conceptual stochastic climate models," Stochastics and Dynamics, vol. 2, pp. 311-326, 2002.

[12] Y. Wang, L. Liu, and Y. Wu, "Positive solutions for a nonlocal fractional differential equation," Nonlinear Analysis. Theory, Methods \& Applications, vol. 74, no. 11, pp. 3599-3605, 2011.

[13] H. Crauel and F. Flandoli, "Attractors for random dynamical systems," Probability Theory and Related Fields, vol. 100, no. 3, pp. 365-393, 1994.

[14] H. Crauel, A. Debussche, and F. Flandoli, "Random attractors," Journal of Dynamics and Differential Equations, vol. 9, no. 2, pp. 307-341, 1997.

[15] B. Wang, "Random attractors for the stochastic BenjaminBONa-Mahony equation on unbounded domains," Journal of Differential Equations, vol. 246, no. 6, pp. 2506-2537, 2009.

[16] B. Wang, "Random attractors for the stochastic FitzHughNagumo system on unbounded domains," Nonlinear Analysis. Theory, Methods \& Applications, vol. 71, no. 7-8, pp. 2811-2828, 2009.

[17] D. Li, Y. Guo, and B. Guo, "Random attractor and stationary measure for stochastic long-short wave equations," Communications in Mathematical Sciences, vol. 13, no. 2, pp. 539-555, 2015.

[18] H. Lu and J. Xin, "The existence of a global attractor for the $(2+1)$-dimensional long wave-short wave resonance interaction equation," Nonlinear Analysis. Theory, Methods \& Applications, vol. 73, no. 12, pp. 3767-3778, 2010.

[19] B. Zhu, L. Liu, and Y. Wu, "Existence and uniqueness of global mild solutions for a class of nonlinear fractional reactiondiffusion equations with delay," Computers \& Mathematics with Applications, 2016.

[20] J. U. Kim, “On the stochastic porous medium equation," Journal of Differential Equations, vol. 220, no. 1, pp. 163-194, 2006.

[21] J.-L. Lions and E. Magenes, Problems aux Limites Non Homogenes et Applications, Dunod, Paris, France, 1968.

[22] G. da Prato and J. Zabczyk, Stochastic Equations in Infinite Dimensions, vol. 44, Cambridge University Press, Cambridge, UK, 1992.

[23] R. Temam, Infinite-Dimensional Dynamical Systems in Mechanics and Physics, vol. 68 of Applied Mathematical Sciences, Springer, New York, NY, USA, 2nd edition, 1997.

[24] G. Da Prato and J. Zabczyk, Ergodicity for Infinite Dimensional System, vol. 229, Cambridge University Press, Cambridge, UK, 1996. 


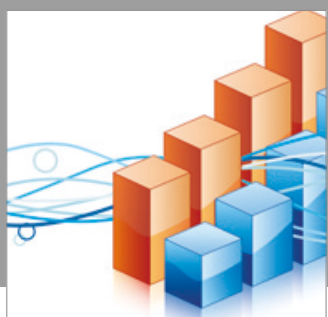

Advances in

Operations Research

vatersals

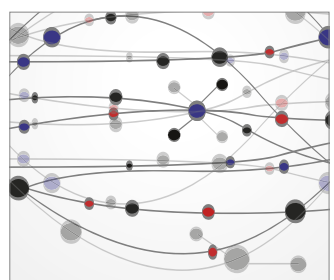

\section{The Scientific} World Journal
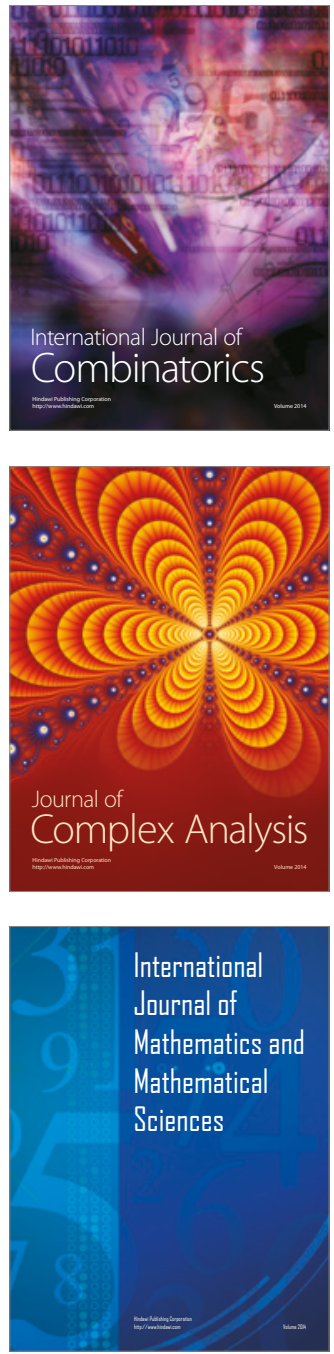
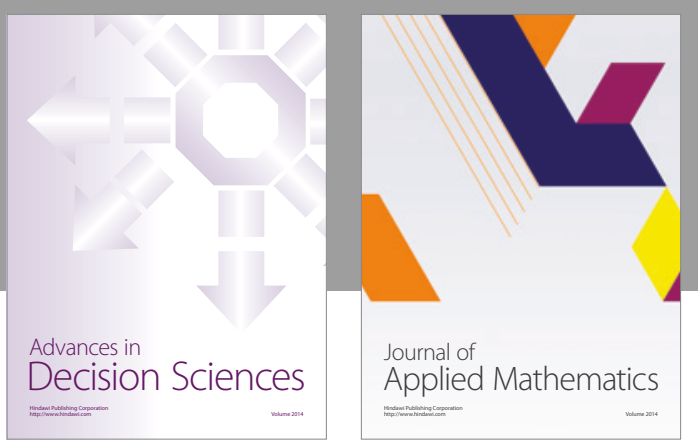

Algebra

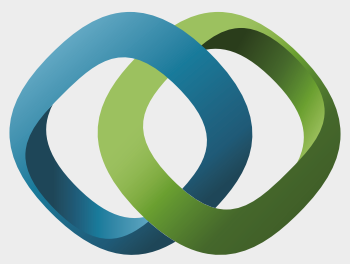

\section{Hindawi}

Submit your manuscripts at

https://www.hindawi.com
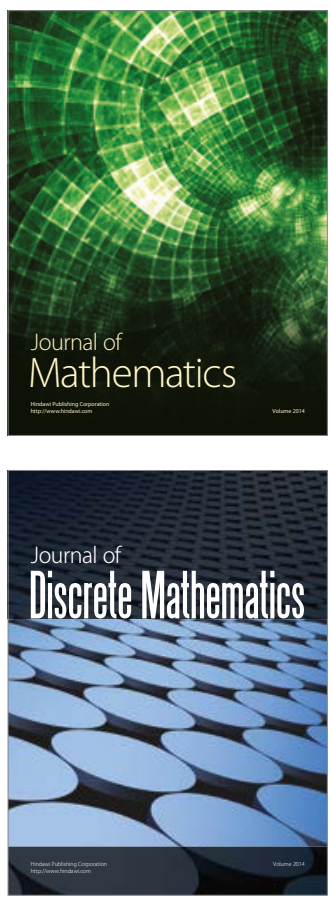

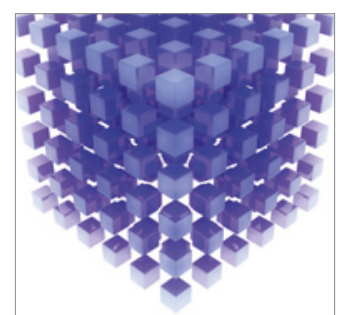

Mathematical Problems in Engineering
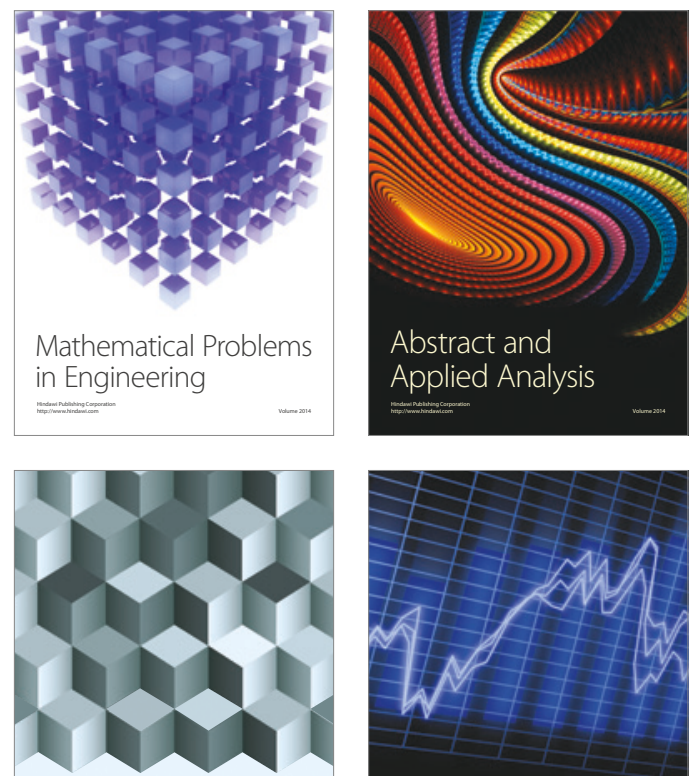

Journal of

Function Spaces

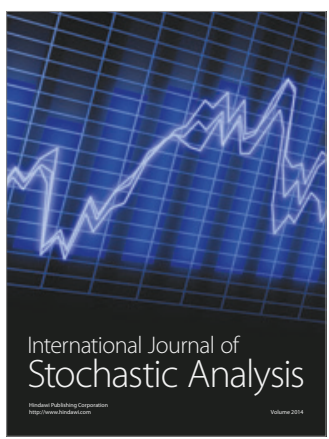

Probability and Statistics
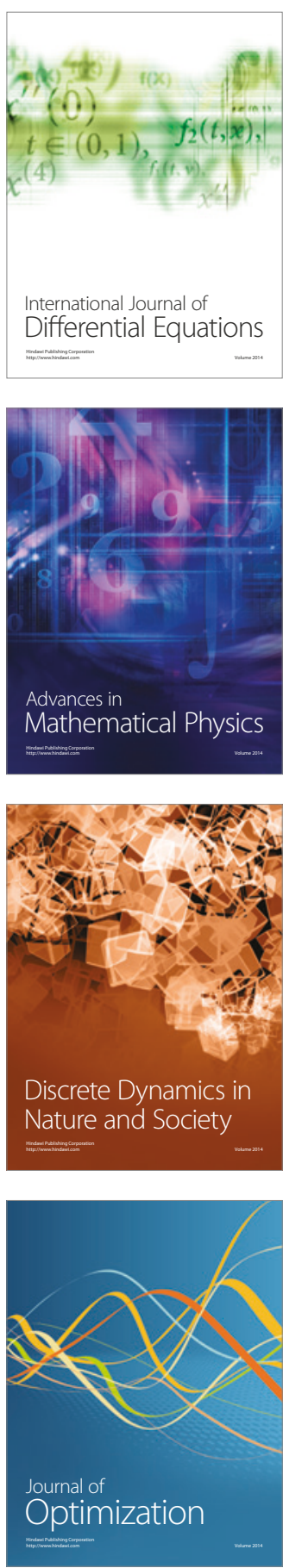